\title{
Heterogeneous tissue distribution of a mitochondrial DNA polymorphism in heteroplasmic subjects without mitochondrial disorders
}

Elmar Kirches, Matthias Michael, Michaela Warich-Kirches, Thomas Schneider, Serge Weis, Guido Krause, Christian Mawrin, Knut Dietzmann

\begin{abstract}
Context-Several maternally inherited point mutations of the mitochondrial genome cause mitochondrial disorders, but the correlation between genotype and phenotype remains obscure in many cases. The same mutation may cause various diseases, probably because of a different tissue distribution.

Objective-To assess the role of random somatic segregation in generating interperson differences by analysis of an apparently neutral polymorphism.

Design-Screening of 81 brain samples from subjects without mitochondrial disorders and selection of five necropsy cases showing a high level of heteroplasmy for the polymorphism.

Main outcome measures-A proportion of various distinct genotypes in the mtDNA pool of the tissues, identified by fluorescent PCR products, representing a short polycytosine tract of variable length in the mitochondrial displacement loop.

Results-Differences were found between organs or groups of organs within subjects, pointing towards somatic segregation of mtDNA. In addition, marked differences of this organ distribution occurred between subjects, which cannot be explained by tissue specific selection.

Conclusions-The observed interperson differences can be explained by somatic segregation, which occurs randomly at various developmental stages. Besides tissue specific selection, this process might participate in the distribution of pathogenic mtDNA mutations. (F Med Genet 2001;38:312-317)
\end{abstract}

Keywords: mtDNA; polymorphism; HVR2; heteroplasmy

The mitochondrial genome (mtDNA) is a double stranded circular DNA $16.6 \mathrm{~kb}$ in length, which encodes 22 tRNAs, two rRNAs, and 13 polypeptides of the oxidative phosphorylation (OXPHOS) complexes. ${ }^{1}$ The displacement loop (D loop) is the only major non-coding segment of mtDNA. Besides important elements for replication and transcription, it contains two hypervariable regions, designated HVR1 and HVR2, which have been widely used in evolutionary studies and population genetics ${ }^{2}$ and, recently, have received considerable attention in the field of forensic medicine. ${ }^{34}$

Since 1988, an increasing number of mtDNA mutations have been described, which are associated with either maternally inherited or sporadic disorders. ${ }^{56}$ Some classical mitochondrial diseases, like myoclonic epilepsy and ragged red fibres (MERRF) ${ }^{7}$ or mitochondrial encephalomyopathy with lactic acidosis and stroke-like episodes (MELAS), ${ }^{8}$ are caused by point mutations in tRNAs. All these defects are maternally inherited, since only mtDNA derived from the oocytes is transmitted to the embryo. Large deletions or duplications in the range of several $\mathrm{kb}$ are typically observed in chronic progressive external ophthalmoplegia (CPEO), in Kearns-Sayre syndrome (KSS), and Pearson's bone marrow syndrome, which are usually sporadic diseases. ${ }^{5}$ Most of these mutations are heteroplasmic, that is, both mutant and wild type DNA coexist in the same person.

Mitochondrial disorders can show considerable variation in the severity of symptoms, and the same mutation may cause a large variety of diseases. The MELAS syndrome may be taken as an example. The severity of the syndrome can vary greatly within a maternal lineage and only severely affected family members may develop full blown symptoms of the central nervous system. Furthermore, the most common mutation, the base substitution A3243G, can sometimes cause different and less severe disorders like CPEO or maternally inherited diabetes with deafness (MIDD). It may cause MELAS/KSS overlap syndromes or neurogenic muscle weakness, ataxia, and retinitis pigmentosa (NARP). These and other examples of phenotypic diversity can be found in the MITOMAP database (http://infinity.gen.emory.edu/mitomap.html) and in the review by Zeviani et al. ${ }^{6}$

It is widely accepted that the percentage of mutant mtDNA in the affected tissues controls the severity of the phenotype in many disorders, but it is more difficult to explain why the same mutation can cause a variety of distinct diseases. It is believed that the heterogeneous tissue distribution might cause this variability. Differences in the percentage of mutant mtDNA distributed between tissues of a subject could be explained by tissue specific selective pressure. However, the strong interperson differences of the tissue distribution of 
Mitochondrial control region

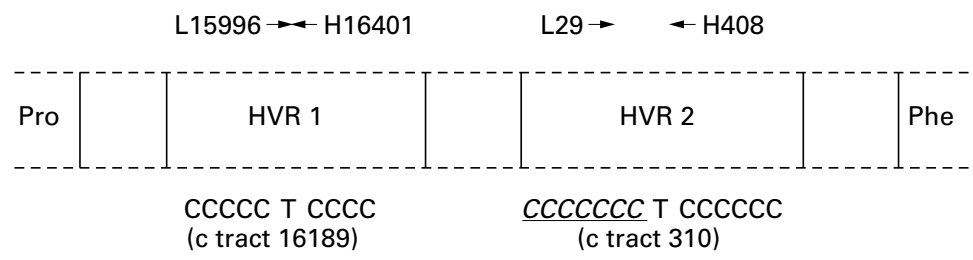

Figure 1 Diagram showing the mitochondrial control region between the $R$ RAs proline (Pro) and phenylalanine (Phe) of the mitochondrial genome. The numbers refer to the position of primers allowing the amplification of the complete hypervariable regions 1 and 2 (HVR1, HVR2) and to the positions of the thymine residues within the two incomplete c-tracts. The variable first part of the HVR2 c-tract investigated in this study (underlined) extends from $n p 303$ of the human mtDNA to $n p$ 309. The tract of cytosine residues following the thymine in position 310 usually contains six cytosines in contrast to the originally published sequence.

inherited point mutations cannot be explained without the participation of random segregation of heteroplasmic mtDNA genotypes during embryogenesis and/or effects of nuclear background. Although random segregation is widely hypothesised, to our knowledge it has never been shown in human necropsy material using neutral polymorphisms in non-diseased subjects. In this study, we show a high degree of heteroplasmy in a polycytosine tract (c-tract) of the mitochondrial HVR2 region (fig 1 ) in postmitotic tissues ( 81 brain samples). In a second step, we used this marker to analyse the tissue distribution of c-tract variants in various organs of five necropsy cases with a high level of heteroplasmy.

\section{Materials and methods}

SAMPLING AND STANDARD DNA ISOLATION

Fifty five brain samples were derived by laser microdissection from the tumour margin of paraffin embedded surgical astroglioma samples. The samples had originally been obtained during screening for the detection of somatic mutations in these tumours. The cases were selected only when tumour free normal brain parenchyma could be isolated by laser microdissection from the paraffin slides. Fifty two cases were glioblastomas, while three patients suffered from low grade astrocytomas. Laser microdissection of $0.03 \mathrm{~mm}^{2}$ areas from $5 \mu \mathrm{m}$ thick slides was performed by an experienced neuropathologist to exclude contamination of the brain tissue samples with tumour tissue. Furthermore, areas with necrosis or other microscopically visible alterations as well as blood vessels were excluded by this technique. Twenty six necropsied brains and other organs of seven of these 26 patients were derived from subjects who had suffered from various diseases, but not mitochondrial disorders. In the necropsy cases, the tissue was taken from a 1.5 $\mathrm{cm}^{2}$, dewaxed, $10 \mu \mathrm{m}$ thick paraffin section followed by proteinase $\mathrm{K}$ digestion, standard phenol/chloroform extraction, and ethanol precipitation of DNA. ${ }^{9}$. The dried DNA pellet was dissolved in $30 \mu \mathrm{l}$ TE buffer. Five $\mu \mathrm{l}$ of this solution were used for subsequent PCR reactions. A serial control section had been stained in each case with haematoxylin and eosin and was studied by an experienced neuropathologist. The organs examined are listed in fig 2. Both sexes were represented in the study ( $53 \%$ male and $47 \%$ female, $n=81$ ) and the ages ranged from 8 to 89 years.

\section{LASER MICRODISSECTION}

Four $\mathrm{cm}^{2}$ pieces of a $1.3 \mu \mathrm{m}$ polyethylene membrane (LPC membrane, PALM, Bernried, Germany) were mounted on cover slides. Five $\mu \mathrm{m}$ thick paraffin sections were placed on the poly-L-lysine treated membranes and incubated overnight at $37^{\circ} \mathrm{C}$, followed by $\mathrm{H} \& \mathrm{E}$ staining (two minutes Mayer's haemalaun, one minute $2 \%$ eosin). After drying at room temperature overnight, small circles of 0.03 $\mathrm{mm}^{2}$ were microdissected by laser pressure capturing (LPC) using a Robot Microbeam System (PALM, Bernried, Germany). The dissected material was treated with $30 \mu \mathrm{l}$ of $1 \times$ PCR buffer containing $200 \mu \mathrm{g} / \mathrm{ml}$ proteinase $\mathrm{K}$, digested for two hours at $55^{\circ} \mathrm{C}$, and incubated for 10 minutes at $94^{\circ} \mathrm{C}$. Ten $\mu$ l of this solution were used for subsequent PCR reactions.

PCR ANALYSIS OF FRAGMENT LENGTH

Ten $\mu l$ of the crude DNA extracts from microdissected samples or $5 \mu \mathrm{l}$ of conventionally prepared DNA from single paraffin sections diluted with $5 \mu \mathrm{TE}$ buffer were used for subsequent PCR reactions. Goldstar-polymerase (Eurogentec, Belgium) (0.5 units) and all other PCR components were added in a total volume of $40 \mu 11 \times$ PCR buffer. PCR cycle conditions were one minute at $94^{\circ} \mathrm{C}$, one minute at $58^{\circ} \mathrm{C}$, and one minute at $72^{\circ} \mathrm{C}$ (35 cycles). The 6-FAM 5' labelled forward primer was a $20 \mathrm{bp}$ sequence starting at $\mathrm{np} 266$ of $\mathrm{mtDNA}^{1}$ and the 20 bp reverse primer started at np 427 . After controlling the products on silver stained PAGE gels, the samples were digested with HaeIII, which creates a blunt end at np 333 immediately behind the c-tract of interest. Using cloned and sequenced HVR2 fragments from an earlier study ${ }^{10}$ and known HVR2 blood sequences, we could clearly determine the absolute c-tract length on a 373A sequencer (Applied Biosystems, Foster City, CA) by comparison with known sequences. Mixing experiments of known variants showed that a certain proportion of two variants always resulted in the same proportion of both peaks in the sequencing gels. Furthermore, a fraction of $10 \%$ of a minor variant with a length difference of $1 \mathrm{bp}$ could be detected as a distinct peak. For peak recognition and determination of peak amplitudes, the GeneScan system from Applied Biosystems was used. The procedure for fragment length analysis is a simplified non-radioactive version of the trimmed PCR method introduced by Marchington et $a l^{11}$ and is less sensitive in the detection of low levels of heteroplasmy. However, the sensitivity was sufficient for the purposes of this study.

\section{Results}

The highly sensitive analysis of the c-tract length allowed the detection of heteroplasmy in 54 of the 81 brain samples analysed $(67 \%)$. In five selected necropsy cases with a high level of heteroplasmy in the brain, several additional organs were examined. Fig 2 shows the electrophoretic peak patterns obtained from 
A

Patient 1
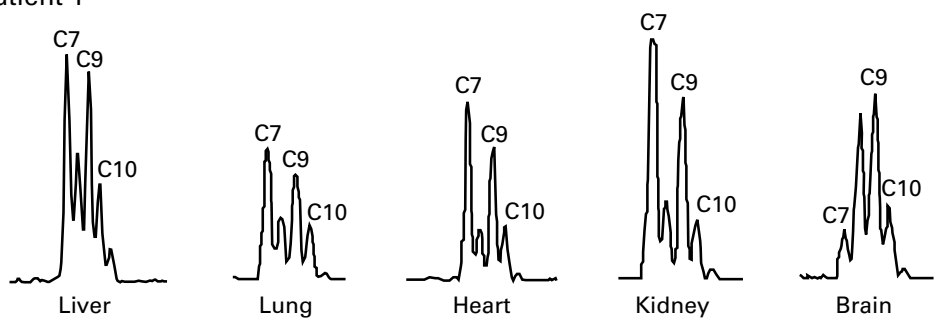

Patient 2
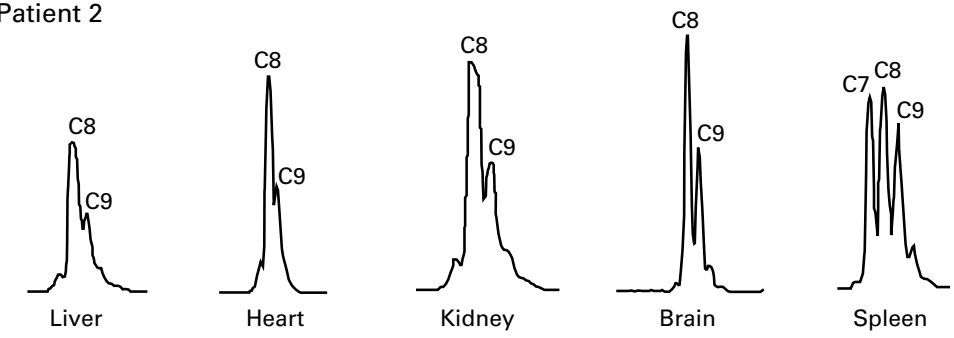

Patient 3
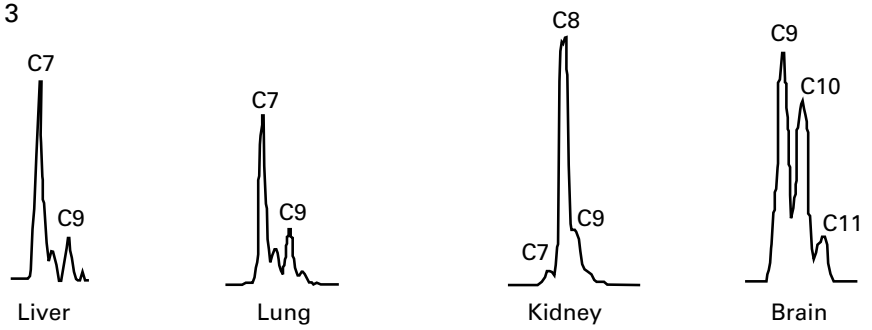

B

Patient 4
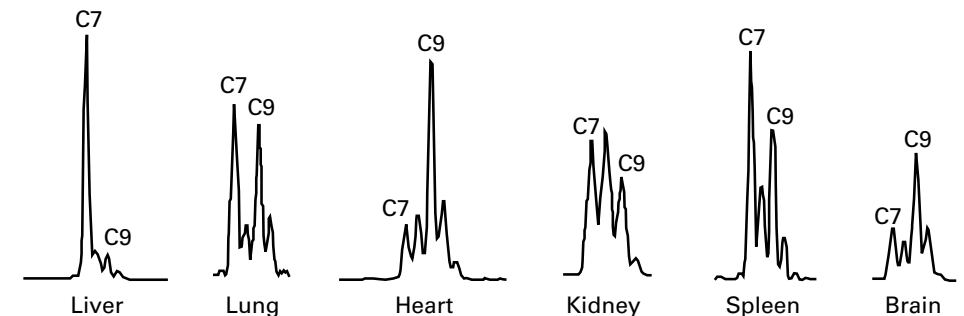

Patient 5
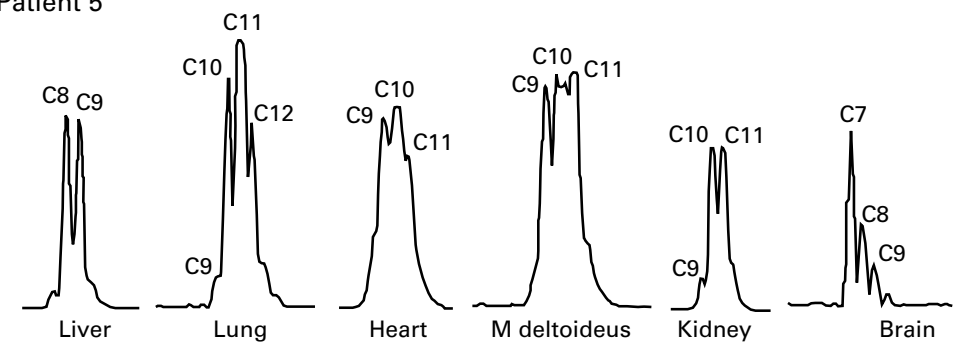

Figure 2 Electropherograms obtained during fragment length analysis of the mitochondrial HVR2 c tract in various organs of five patients with a high level of heteroplasmy. In three subjects $(A)$, the quantitative peak pattern was well conserved among various organs, while it was not in two others (B). Patient 1: female, 65 years. Patient 2: male, 39 years. Patient 3: female, 66 years. Patient 4: male, 34 years. Patient 5: female, 61 years.

these tissues. Each peak in these electropherograms represents an mtDNA clone with a certain c-tract length. Two neighbouring peaks differ by $1 \mathrm{bp}$. While the absolute amplitudes depend on various experimental parameters (such as the amount of DNA in the sample), the relative amplitudes (peak ratios) remain unchanged in a constant proportion of different mtDNAs. This fact had been checked by mixing experiments with cloned DNA. Although the amplitude ratios are not numerically identical to the proportions of the corresponding mtDNA clones, they were used to define the peak ratios in table 1 , since amplitudes can be measured more precisely than the peak areas. According to the electrophoretic mobility of cloned and sequenced reference DNA in the gels, the peaks in the diagrams could be labelled with their absolute c-tract length. It is known ${ }^{12}$ and is supported by our own sequencing data ${ }^{10}$ that usually only the first half of the incomplete HVR2 c-tract exhibits length variability (fig 1 ). Therefore, the peak designations in fig 2 reflect only the number of cytosine residues within this region.

In patient 1 , the same five clones could be identified in all organs examined, but their ratios differed strikingly between the brain and the four body organs (fig 2A). Table 1 shows a detailed analysis of peak ratios of the four major genotypes of this patient, which were significantly above background in all organ samples (C7, C8, C9, and C10). It can be seen that all three ratios differed by less than $13 \%$ within the two endodermal derivatives, that is, lung and liver, and by less than $15 \%$ between the two mesodermal derivatives, that is, heart and kidney. On the other hand, some of the peak ratios differed up to 1.9 -fold between endodermal and mesodermal derivatives, up to 6.8-fold between brain (neuroectoderm) and endodermal derivatives and up to 12.5 -fold between brain and mesodermal derivatives.

When the peak ratio was calculated for the two dominant peaks in patient 2 (C8, C9), it differed by less than $11 \%$ in the liver, kidney, heart, and brain. The peak ratios between these organs and spleen differed by the factor 1.5. In addition, the high amplitude of the $\mathrm{C} 7$ peak in the spleen separated this organ from the others, in which this clone was barely detectable (fig 2A).

In patient 3, the peak ratios $\mathrm{C} 7 / \mathrm{C} 8$ and C7/C9 differed by $23 \%$ and 3\%, respectively, between the two endodermal derivatives, lung and liver, while the brain (neuroectoderm) and kidney (mesoderm) showed qualitatively different patterns (fig $2 \mathrm{~A}$ ). All tissues from patient 4 contained the same four c-tract genotypes, but in highly varying proportions, while in patient 5 qualitative/quantitative differences occurred between all tissues (fig 2B). In the deltoid muscle of patient 5, one peak could not be attributed with sufficient precision to a certain length variant in repeated measurements. In fig $2 \mathrm{~B}$, this peak was designated $\mathrm{C} 10$, although it seemed to consist of two independent variants with similar electrophoretic mobility.

To elucidate the stability of the HVR2 c tract further, we analysed in brain tissue of two cases without heteroplasmy (homoplasmic controls) the cerebral cortex (temporal and occipital lobes), cerebellar cortex, kidney, liver, lung, heart, skeletal muscle, spleen, colon, and oesophagus. In all the tissues of these two cases (a 63 year old male and a 89 year old female) 
Table 1 Peak ratios in various organs of patients 1, 2, and 3

\begin{tabular}{|c|c|c|c|}
\hline Organ & Peaks & Amplitude ratio & Fetal tissue \\
\hline \multicolumn{4}{|c|}{ Patient 1 (F, 65 years) } \\
\hline Liver & $\mathrm{C} 7 / \mathrm{C} 8$ & 1.78 & Endoderm \\
\hline Lung & $\mathrm{C} 7 / \mathrm{C} 8$ & 2.00 & Endoderm \\
\hline Heart & $\mathrm{C} 7$ / C8 & 3.64 & Mesoderm \\
\hline Kidney & $\mathrm{C} 7 / \mathrm{C} 8$ & 3.36 & Mesoderm \\
\hline Brain & $\mathrm{C} 7 / \mathrm{C} 8$ & 0.28 & Neuroectoderm \\
\hline Liver & C7 / C9 & 1.06 & Endoderm \\
\hline Lung & $\mathrm{C} 7$ / C9 & 1.18 & Endoderm \\
\hline Heart & $\mathrm{C} 7$ / C9 & 1.33 & Mesoderm \\
\hline Kidney & $\mathrm{C} 7$ / C9 & 1.33 & Mesoderm \\
\hline Brain & $\mathrm{C} 7$ / C9 & 0.26 & Neuroectoderm \\
\hline Liver & $\mathrm{C} 7$ / C10 & 2.36 & Endoderm \\
\hline Lung & C7 / C10 & 2.42 & Endoderm \\
\hline Heart & $\mathrm{C} 7 / \mathrm{C} 10$ & 3.40 & Mesoderm \\
\hline Kidney & $\mathrm{C} 7$ / C10 & 3.90 & Mesoderm \\
\hline Brain & $\mathrm{C} 7$ / C10 & 0.64 & Neuroectoderm \\
\hline \multicolumn{4}{|c|}{ Patient 2 (M, 39 years) } \\
\hline Liver & $\mathrm{C} 8$ / C9 & 1.92 & Endoderm \\
\hline Heart & $\mathrm{C} 8$ / C9 & 2.02 & Mesoderm \\
\hline Kidney & $\mathrm{C} 8 / \mathrm{C} 9$ & 1.88 & Mesoderm \\
\hline Brain & $\mathrm{C} 8$ / C9 & 1.83 & Neuroectoderm \\
\hline Spleen ${ }^{\star}$ & $\mathrm{C} 8 / \mathrm{C} 9$ & 1.29 & Mesoderm \\
\hline \multicolumn{4}{|c|}{ Patient 3 ( $F, 66$ years) } \\
\hline Liver & $\mathrm{C} 7$ / C8 & 4.40 & Endoderm \\
\hline Lung & C7 / C8 & 4.53 & Endoderm \\
\hline Kidney† & $\mathrm{C} 7$ / C8 & 0.03 & Mesoderm \\
\hline Braint & C7 not detectable & 0 & Neuroectoderm \\
\hline Liver & C7 / C9 & 3.41 & Endoderm \\
\hline Lung & $\mathrm{C} 7$ / C9 & 2.72 & Endoderm \\
\hline Kidney & $\mathrm{C} 7$ / C9 & 0.13 & Mesoderm \\
\hline Brain & C7 not detectable & 0 & Neuroectoderm \\
\hline
\end{tabular}

Mean peak ratios were determined in two measurements.

${ }^{\star}$ In the spleen of patient 2, a major C7 peak was visible in addition, which was difficult to detect in the other organs (see fig $2 \mathrm{~A}$ ).

tQualitative differences were also detected between kidney and brain of patient 3 (see fig $2 \mathrm{~A}$ ).

only one identical c-tract genotype (C7) was detected.

\section{Discussion}

RELEVANCE OF THE HVR2 C-TRACT IN THE ANALYSIS OF MTDNA SEGREGATION IN OOCYTES AND TISSUES

A genetic bottleneck occurring during mtDNA germline transmission in mammals is an important concept in mitochondrial genetics for explaining rapid fixation of mutations. A complete switch to another homoplasmic genotype between mother and offspring has been observed in Holstein cows. ${ }^{13}$ Furthermore, estimates for the number of segregating units constituting the mtDNA pool of the offspring showed a strong variation depending on the species investigated and the mathematical models used. ${ }^{14-18}$ The possibility of a narrow bottleneck in humans would provide an explanation for the rapid acquisition of high mutational loads, which can sometimes be observed during transmission of mitochondrial disorders. In a female KSS patient with multiple mtDNA rearrangements including duplications, a postmortem analysis of the ovary was performed by Marchington et al. ${ }^{19}$ Patients with duplications may have a propensity for germline transmission. ${ }^{20}$ Rearranged molecules were detected in the germline of the KSS case, but the percentage of mutant DNA varied markedly among 15 oocytes.

Moreover, Marchington et $a l^{11}$ showed that the length of the HVR2 c-tract is a polymorphic marker, which can be used to determine differences in the mtDNA pools in mature human oocytes in any female. This method allowed a direct analysis of heteroplasmic mtDNA genotypes in the germline of normal subjects without mitochondrial disorders, and supported the occurrence of an early genetic bottleneck during oocyte maturation. In that study, some mature oocytes were found to be heteroplasmic in their HVR2 c-tract. This suggests that heteroplasmy in this region should sometimes be transmitted to the offspring. Therefore, the tissue distribution of this marker in some selected subjects with a high level of heteroplasmy provides a source of information about the fate of transmitted mtDNA during embryogenesis and further development. A prerequisite would be sufficient stability of the marker (low level of somatic mutations). This stability was tested in our study by analysing the two elderly homoplasmic subjects (63 and 89 years), showing the same homoplasmic genotype in all 11 tissues examined, which is in accordance with the results of Marchington et $a l^{11}$ obtained from two to five organs from five necropsy cases. ${ }^{11}$

TISSUE DISTRIBUTION OF MUTATIONAL LOAD AND SELECTIVE PRESSURE

The segregation of mtDNA within the mother's germline can be one factor responsible for the unpredictable level of heteroplasmy transmitted to the offspring. It can therefore partly explain the marked differences in phenotypic severity sometimes observed in a maternal lineage. The specific tissue distribution of mutations found in a certain disorder may be explained by the varying energy demands, differences between proliferating and postmitotic cells, and other factors, which might cause a tissue specific selective pressure. The accumulation of mtDNA deletions in muscle tissue of CPEO or KSS patients might be explained by a replicative advantage of shorter molecules in postmitotic tissues, where the disturbed mitochondria lead to no essential disadvantage for cell survival. On the other hand, deletions might be eliminated from rapidly dividing haematopoetic stem cells by negative selective pressure at the cellular level, since mitochondrial dysfunction may hamper cell division. Somatic segregation might lead to high levels of mutant mtDNA in some stem cells and low levels in others. Cells with a high mutational load may stop to divide. The positive selection of deleted molecules in postmitotic tissues might explain the accumulation of deletions in skeletal muscle over time associated with progression of disease. ${ }^{21}$

The pathogenic transition A3243G decreases over time in the blood, ${ }^{22}$ probably because the mitochondrial dysfunction hampers cell division. At first sight, a transition should confer no replicative advantage to the mtDNA molecules carrying it. Therefore, an increase over time in the mutational load of a point mutation in skeletal muscle ${ }^{23}$ seems more difficult to explain than an accumulation of deleted molecules. However, cell culture experiments with cybrids carrying the transition 
A3243G showed a marked replicative advantage. ${ }^{24}$ Moreover, not all pathogenic point mutations show similar behaviour. The transversion T8993G is not elimininated from blood. Generally, substitutions at nucleotide position 8993 were found at uniformly high heteroplasmic levels in all tissues examined so far. ${ }^{25}$

Tissue specific selection cannot explain how marked differences in the tissue distribution of a base substitution might occur, leading to a variety of different diseases. In addition, not all pathogenic mutations are prone to selective pressure. $^{25}$

\section{RANDOM SEGREGATION VERSUS TISSUE SPECIFIC} SELECTION

In our study, nearly identical quantitative patterns of the heteroplasmic HVR2 c-tract were detected within the organ pairs lung/liver (endoderm) and heart/kidney (mesoderm) in a 65 year old woman (patient 1 in fig $2 \mathrm{~A}$, table 1 ), while the pattern differed markedly in all three germinal layers (endoderm, mesoderm, exoderm). The differences between the organ groups can be explained by random segregation of mtDNA. It is most likely that the process occurred at early stages of embryogenesis. This could explain the unequal distribution of the different genotypes to the three germinal layers in this case. In patient 2, four organs had a similar quantitative peak pattern, while the spleen had a different one. This result and the complex patterns observed in the other patients are compatible with random somatic segregation of mtDNA, which is not coupled to distinct stages of embryogenesis.

The variability of the HVR2 c-tract is a polymorphism, which is thought to confer no selective advantage or disadvantage to the cells carrying different genotypes. Nevertheless, a possible role of selection for the tissue distribution of this marker has to be discussed.

Surprisingly, some mtDNA polymorphisms in mammals seem to be not truly neutral and a tissue specific selection for some polymorphisms seems to occur. Jenuth et $a l^{26}$ derived heteroplasmic mice with a hybrid mitochondrial genome containing mtDNA from the mouse strains BALB/cBy1 (BALB) and NZB/ BINJ (NZB). An analysis of the tissue distribution of both genomes in the hybrid mice showed a decrease of the NZB genotype over one year postpartum in the blood and spleen and an increase in kidney and liver, while in several other tissues no significant changes over time could be observed. Furthermore, the nuclear background (BALB or BALB/NZB) did not influence the distribution of the mitochondrial genomes.

The occurrence of a tissue specific selection for polymorphisms was clearly established by these experiments, although the mechanism remains unknown. Differences in replicative properties caused by sequence variation in the D loop were unlikely, since the genomes did not differ in the conserved sequence elements. Another speculative explanation, suggested by the authors, is a dependence of mitochondrial turnover on mtDNA genotype. At least a selection at the cellular level could occur, based on mitochondrial function. Most mitochondrial proteins are encoded by nuclear genes. Since tissue specific differences in such proteins might exist, some of these isoforms might cooperate more effectively with certain isoforms of mtDNA encoded proteins.

In our study, a highly variable tissue distribution was found for a polymorphic marker in five people. Although compatible with random somatic segregation, this situation cannot be explained by tissue specific selection. In patient 1 , the peak ratio $\mathrm{C} 7 / \mathrm{C} 8$ was much higher in kidney, when compared with liver or lung, while the situation was reversed in patients 3 and 4 (table 1, fig 2B). In patient 2, the ratio $\mathrm{C} 7 / \mathrm{C} 8$ was quite similar in kidney and liver (table 1). Nevertheless, the occurrence of selection is not ruled out by these results. Regarding the study of Jenuth et $a l,{ }^{26}$ it seems possible that in some patients additional polymorphisms in mtDNA coding regions are coupled with certain c-tract genotypes, thereby providing a basis for selection in some organs.

Taken together, our results suggest that different mtDNA clones, which have passed the bottleneck in the female germline can be distributed in varying proportions to various organs during embryogenesis leading to a strong imbalance in the composition of mtDNA pools among organs. Marked interperson differences will result from the fact that this process occurs randomly during embryogenesis without being associated with certain developmental stages or tissues.

We gratefully acknowledge the excellent technical assistance of Mrs I Schellhase, G Küchler, I Otto, and S Hartmann. We thank Dr R Schneider-Stock and Prof Dr A Roessner (Magdeburg) for the use of the microdissection device. This work was supported by the State of Sachsen Anhalt, Contract Grant Sponsor $2526 \mathrm{~A} / 0086 \mathrm{H}$ and by the Deutsche Forschungsgemeinschaft, Contract Grant Sponsor KI 530/2-1.

1 Anderson S, Bankier AT, Barell BG, de Bruijn MHL, Coulson AR; Drouin J, Eperon IC, Nierlich DP, Roe BA, Sanger F, Schreier PH, Smith AJH, Staden R, Young IG. Sequence and organization of the human mitochondrial genome. Nature 1981;290:457-64.

2 Stoneking M, Hedgecock D, Higuchi RG, Vigilant L, Erlich HA. Population variation of human mtDNA control region sequences detected by enzymatic amplification and sequence-specific oligonucleotide probes. Am f Hum Genet 1991;48:370-82.

3 Grzybowski T. Extremely high levels of human mitochondrial DNA heteroplasmy in single hair roots. Electrophoresis 2000;21:548-53.

4 Calloway CD, Reynolds RL, Herrin GL, Anderson WW. The frequency of heteroplasmy in the HV II region of mtDNA differs across tissue types and increases with age. Am f Hum Genet 2000;66:1384-97.

5 Wallace DC. Diseases of the mitochondrial DNA. Anпи Rev Biochem 1991;61:1175-212.

6 Zeviani M, Tiranti V, Piantadosi C. Mitochondrial disorders. Rev Mol Med 1998;77:59-72.

7 Shoffner JM, Lott MT, Lezza AM, Seibel P, Ballinger SW, Wallace DC. Myoclonic epilepsy and ragged-red fiber disease (MERRF) is associated with a mitochondrial DNA tRNA (Lys) mutation. Cell 1990;61:931-7.

8 Enter C, Müller-Höcker J, Zierz S, Kurlemann G, Pongratz D, Forster C, Obermeier- Kusser B, Gerbitz KD. A specific point mutation in the mitochondrial genome of Caucasians with MELAS. Hum Genet 1991;88:233-6.

9 Sambrook J, Fritsch EF, Maniatis T. Molecular cloning. A laboratory manual. New York: Cold Spring Harbor Laboratory Press, 1989.

10 Kirches E, Michael M, Woy C, Schneider T, Warich-Kirches M, Schneider-Stock R, Winkler K, Wittig H, Dietzmann K. Loss of heteroplasmy in the displacement loop of brain mitochondrial DNA in astrocytic tumors. Genes Chrom Cancer 1999;26:80-3.

11 Marchington DR, Hartshorne GM, Barlow D, Poulton J. Homopolymeric tract heteroplasmy in mtDNA from tissues and single oocytes: support for a genetic bottleneck. Am f Hum Genet 1997;60:408-16. 
12 Hauswirth WW, Clayton DA. Length heterogeneity of a conserved displacement-loop sequence in human mito-

13 Koehler CM, Lindberg GL, Brown DR, Beitz DC, Freeman AE, Mayfield JE, Myers AM. Replacement of bovine mitochondrial DNA by a sequence variant within one 1991;129:247-55.

14 Hauswirth W, Laipis P. Transmission genetics of mammalian mitochondria: a molecular model and experimenta evidence. In: Quagliarello E, ed. Achievements and perspectives of mitochondrial research. Vol 2. Biogenesis. Amsterdam: Elsevier Biomedical, 1985.

15 Bendall KE, Macaulay VA, Baker JR, Sykes BC. Heteroplasmic point mutations in the human mtDNA control region. Am 7 Hum Genet 1996:59:1276-87.

16 Ashley C, Laipis P, Hauswirth W. Rapid sequestration of heteroplasmic bovine mitochondria. Nucleic Acids Res 1989;17:7325-31.

17 Jenuth JP, Peterson A, Fu K, Shoubridge EA. Random genetic drift in the female germline explains the rapid segregation of mammalian mitochondrial DNA. Nat Genet 1996;14:146-51.

18 Solignac M, Genermont J, Monnerot M, Mounolou J. Drosophila mitochondrial genetics: evolution of heteroplasmy through germ line cell divisions. Genetics 1987;117:68796.

19 Marchington DR, Macaulay V, Hartshorne GM, Barlow D, Poulton J. Evidence from human oocytes for a genetic bottleneck in an mtDNA disease. Am f Hum Genet 1998;63: 769-75.

20 Poulton J, Holt I. Mitochondrial DNA: does more lead to less? Nat Genet 1994;8:313-15.

21 Larsson NG, Holme E, Kristiansson B, Oldfors A, Tulinius M. Progressive increase of the mutated mitochondrial DNA fraction in Kearns-Sayre syndrome. Pediatr Res 1990; 28:131-6.

22 't Hart LM, Jansen JJ, Lemkes HH, de Knijff P, Maassen JA. Heteroplasmy levels of a mitochondrial gene mutation associated with diabetes mellitus decrease in leucocyte DNA upon aging. Hum Mutat 1996;7:193-7.

23 Weber K, Wilson JN, Taylor L, Brierley E, Johnson MA, Turnbull DM, Bindoff LA. A new mtDNA mutation showing accumulation with time and restriction to skeletal muscle. Am 7 Hum Genet 1997;60:373-80.

24 Yoneda M, Chomyn A, Martinuzzi A, Hurko O, Attardi G. Marked replicative advantage of human mtDNA carrying a point mutation that causes the MELAS encephalomyopapoint mutation that causes the MELAS encephal
thy. Proc Natl Acad Sci USA 1992;89:11164-8.

25 White SL, Shanske S, McGill J, Mountain H, Geraghty MT, DiMauro S, Dahl HHM, Thorburn DR. MitochonMT, DiMauro S, Dahl HHM, Thorburn DR. Mitochon-
drial DNA mutations at nucleotide 8993 show a lack of trissue- or age-related variation. If Inherit Metab Dis tissue- or age-relatc

26 Jenuth JP, Peterson AC, Shoubridge EA. Tissue-specific selection for different mtDNA genotypes in heteroplasmic mice. Nat Genet 1997;16:93-5.

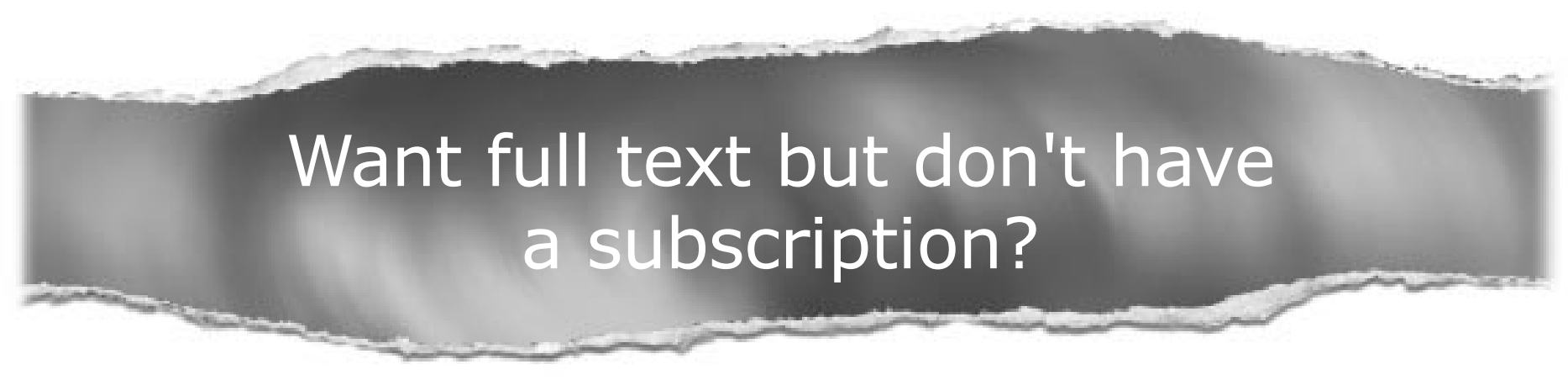

Pay per view

For just $\$ 8$ you can purchase the full text of individual articles using our secure online ordering service. You will have access to the full text of the relevant article for 48 hours during which time you may download and print the pdf file for personal use.

www.jmedgenet.com 